\title{
Stereotactic body radiation therapy for the treatment of pleural metastases in patients with thymoma: a retrospective review of 22 patients
}

\author{
Giulia Pasquini ${ }^{1}$, Claudia Menichelli ${ }^{2}$, Gabriella Pastore ${ }^{3}$, Franco Casamassima ${ }^{2}$, \\ Maria Grazia Fabrini ${ }^{4}$, Sabrina Cappelli ${ }^{5}$, Simona Valleggi ${ }^{5}$, Maurizio Lucchesi ${ }^{5}$, Marco Lucchi ${ }^{6}$, \\ Roberta Ricciardi ${ }^{7}$, Michelangelo Maestri ${ }^{7}$, Melania Guida ${ }^{7}$, Antonio Chella ${ }^{5}$, Iacopo Petrini ${ }^{8}$
}

${ }^{1}$ Medical Oncology, Department of Translational Research and New Technology in Medicine and Surgery, University of Pisa, Pisa, Italy; ${ }^{2}$ Department of Radiotherapy, Institute of Clinical Research Ecomedica, Empoli, Italy; ${ }^{3}$ Department of Medical Physics, Institute of Clinical Research Ecomedica, Empoli, Italy; ${ }^{4}$ Radiotherapy Unit, University Hospital of Pisa, Pisa, Italy; ${ }^{5}$ Pneumology Unit, University Hospital of Pisa, Pisa, Italy; ${ }^{6}$ Thoracic Surgery, Department of Surgical Pathology, Molecular Medicine and Critical Area, University Hospital of Pisa, Pisa, Italy; ${ }^{7}$ Neurology Unit, Department of Clinical and Experimental Medicine, University Hospital of Pisa, Pisa, Italy; ${ }^{8}$ General Pathology, Department of Translational Research and New Technology in Medicine and Surgery, University of Pisa, Pisa, Italy

Contributions: (I) Conception and design: I Petrini, G Pasquini; (II) Administrative support: S Valleggi; (III) Provision of study materials or patients: C Menichelli, F Casamassima, MG Fabrini, S Cappelli, M Lucchesi, M Lucchi, R Ricciardi, M Maestri, M Guida, A Chella, I Petrini; (IV) Collection and assembly of data: G Pasquini; (V) Data analysis and interpretation: I Petrini, G Pasquini; (VI) Manuscript writing: All authors; (VII) Final approval of manuscript: All authors.

Correspondence to: Iacopo Petrini, MD, PhD. General Pathology, Department of Translational Research and New Technology in Medicine, Via Savi 10, Pisa 56126, Italy. Email: iacopo.petrini@unipi.it.

Background: Thymomas can benefit of cytoreductive surgery even if a complete resection is not feasible. The pleural cavity is the most common site of progression and the resection of pleural metastases can be performed in selected patients. We evaluated the results of stereotactic body radiation therapy for the treatment of pleural metastases in patients not eligible for surgery.

Methods: We retrospectively selected 22 patients treated with stereotactic body radiation therapy for pleural metastases between 2013 and 2019. According to RECIST criteria 1.1 modified for thymic epithelial tumors, time to local failure and progression free survival were calculated using Kaplan-Meier method.

Results: The median age was 40 years (range, 29-73 years). There were 1 A, 3 AB, 3 B1, 3 B2, 3 B2/B3 and $9 \mathrm{~B} 3$ thymomas. Pleural metastases and primary tumor were synchronous in 8 patients. Five patients had a single pleural metastatic site and 17 presented multiple localizations. Sixteen patients received stereotactic body radiation therapy on multiple sites of pleural metastases. The median dose of radiation was 30 Gy (range, 24-40 Gy). With a median follow-up of 33.2 months (95\% CI: 13.1-53.3 months), ten patients experienced disease progression with a median progression free survival was 20.4 months (95\% CI: 10.7-30.0 months). The disease control rate was $79 \%$ and $41 \%$ after 1 and 2 years, respectively. Local disease control rate was $92 \%$ and $78 \%$ after 1 and 2 years, respectively. There were not significant differences in progression free survival between patients diagnosed with synchronous and metachronous metastases $(\mathrm{P}=0.477)$, across those treated or not with chemotherapy $(\mathrm{P}=0.189)$ and between those who received or not a previous surgical resection of the pleural metastases $(\mathrm{P}=0.871)$. There were not grade 3-4 toxicities related to the treatment.

Conclusions: Stereotactic body radiation therapy of pleural metastases is feasible and offers a promising local control of diseases. The impact of this treatment on patients' survival is hardly predictable because of the heterogeneous clinical behavior of thymomas.

Keywords: Stereotactic body radiation therapy; pleural metastases; thymoma 
Submitted Nov 22, 2019. Accepted for publication Mar 18, 2020.

doi: 10.21037/jtd-19-3799

View this article at: https://dx.doi.org/10.21037/jtd-19-3799

\section{Introduction}

Thymic epithelial tumors are rare neoplasms with an incidence of 0.32 -case every 100,000 persons/year (1). According to the 2015 WHO schema, thymic epithelial tumors are divided in thymic carcinoma and thymoma which are further classified in $\mathrm{A}, \mathrm{AB}, \mathrm{B} 1, \mathrm{~B} 2$ and $\mathrm{B} 3$ histotypes (2). All thymic epithelial tumors are malignant but thymic carcinomas have a more aggressive behavior with about $50 \%$ of the patients alive after 10 years from the diagnosis (3). Thymomas have variable prognosis being some tumors indolent and others frankly aggressive. Stage at the diagnosis, WHO histotype, completeness of surgical resection and the presence of GTF2I somatic mutation are the major prognostic factors in thymomas $(3,4)$.

Surgery is the mainstay of treatment for localized disease. In locally advanced neoplasms, preoperative chemotherapy with or without radiotherapy plays a role in downsizing the primary tumor and enhances the chance of complete resections (3). Whereas distant metastases are frequent in thymic carcinomas, thymomas more often progress through the infiltration of mediastinal structure and directly disseminate into the pleural cavity (5). The use of surgical debulking even in not completely resectable thymoma is still debated but can be considered for selected patients according to some authors $(6,7)$. Indeed, a meta-analysis suggests that debulking surgery for unresectable thymoma may be associated with improved overall survival (7). The resection of pleural metastases can be considered in selected patients with oligometastatic disease and offers a sufficient local control, postponing the use of chemotherapy $(8,9)$. Cytoreductive surgery of the primary tumor or its pleural metastases can be considered for the treatment of thymomas (6). Surgical resection of pleural metastases is usually not curative and pleural nodules relapse after a variable time interval. For these patients and those not operable for clinical conditions, stereotactic body radiation therapy (SBRT) could be an option for the treatment of pleural metastases.

SBRT is a technique that delivers high doses of radiations in a single treatment or in a small number of fractions with high precision minimizing radiation doses to adjacent normal tissue (10). This technique has been extensively applied in a variety of solid tumors including thoracic malignancies showing a promising local control and relatively few toxicities (11). Currently, there are limited data available regarding the toxicity and the efficacy of SBRT for the treatment of pleural metastases in thymomas. Recently, a cohort study suggests that SBRT is feasible and yields an encouraging local control for the treatment of thymic epithelial tumors including pleural metastases (12). Therefore, we retrospectively review the outcome of patients that received SBRT for the treatment of pleural metastases of thymoma at our institutions.

\section{Methods}

We retrospectively identified 22 thymoma patients treated with SBRT for pleural metastases between 2013 and 2018. All the patients had a histological diagnosis of thymoma and tumors have been classified according to the 2015 WHO classification system (2). Pleural metastases were diagnosed using computer tomography (CT) scans without histological confirmation. The metastatic spread in the pleural cavity was histologically confirmed in 14 patients that underwent surgical resection. We included in this analysis pleural metastases with a thickness measured from the chest wall of at least $10 \mathrm{~mm}$ patients with thymic carcinoma were excluded from the analysis. Stage at the diagnosis was defined according to the Masaoka and Koga system (13). Patient's performance status was defined according to the Eastern Cooperative Group (ECOG) criteria before the beginning of SBRT administration. Objective response and tumor progression was defined according to RECIST 1.1 criteria modified for thymic epithelial tumors (14). Toxicity was graded according to NIH-CTCAE v5. The study was conducted in accordance with the Declaration of Helsinki (as revised in 2013). The study was approved from our institution ethics committee CEAVNO with number ID 15960 and individual consent for this retrospective analysis was waived.

\section{Radiation treatment planning and delivery}

Planning CT scans were acquired with a multislice-CT with intravenous contrast. Patients were placed in the 

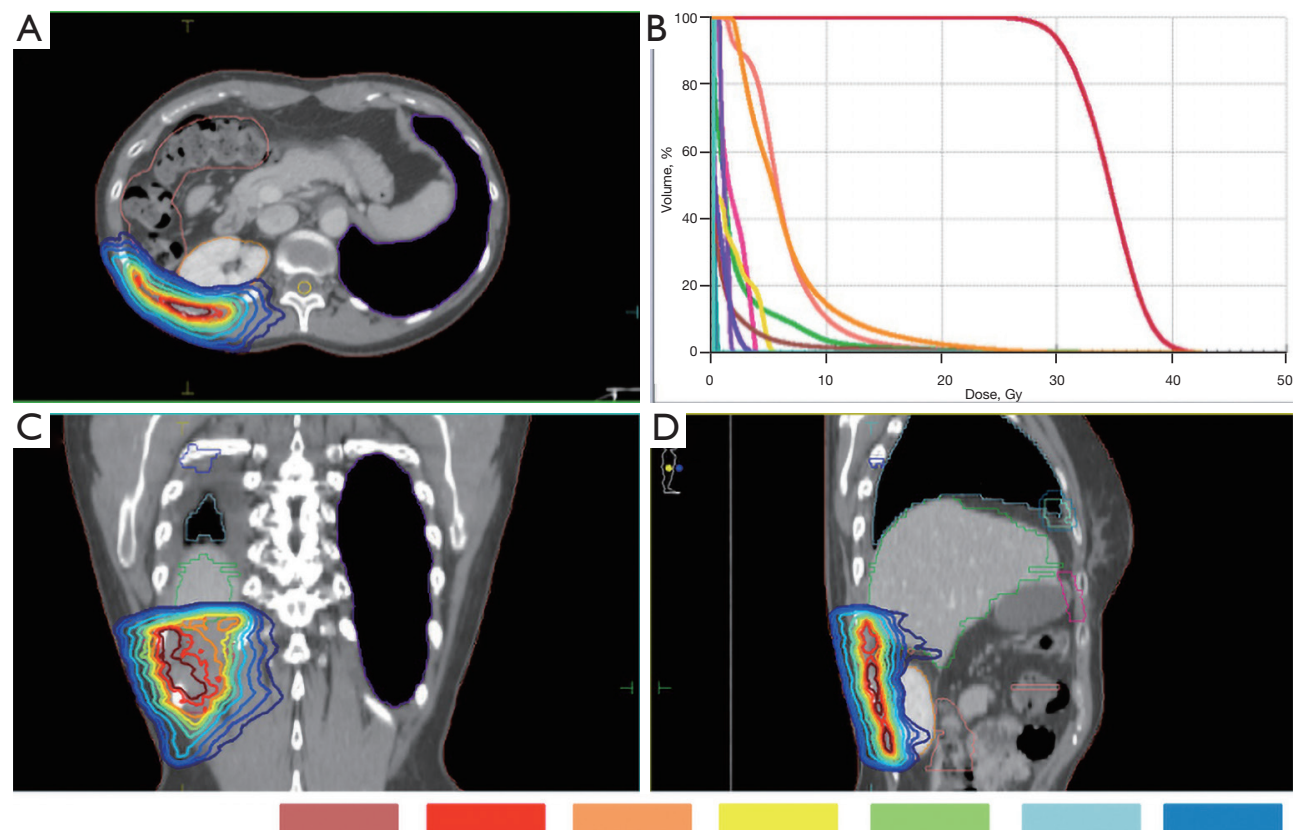

Dose, Gy 37.5 36

Figure 1 Treatment plan and radiation dose distribution for the treatment of a thymoma pleural metastasis, CT reconstruction in transversal plan (A), dose volume histograms (B), CT reconstruction in coronal plan (C), and in sagittal plan (D).

supine position and immobilized with knee-feet device. The planning CT was scanned with $3 \mathrm{~mm}$ slice for tumors that have a diameter more than $3 \mathrm{~cm}$, and with $2 \mathrm{~mm}$ slice for smaller target. A pre-treatment CT was performed and the information was used for gross tumor volume (GTV) definition. Breath-hold technique with the Active Breathing Coordinator system (ABC, Elekta, Cralwley, UK) was used. GTV was delineated in the lung and soft tissue window on CT image. Isotropic expansions of $2 \mathrm{~mm}$ were used for the planning target volume (PTV) delineation. Montecarlo algorithm, including radiobiological models, was employed for volumetric arc therapy treatment plans (Elekta Monaco treatment planning system version 5.11), using coplanar and noncoplanar arcs. SBRT was delivered using $6 \mathrm{MV}$ photons on an Elekta SynergyS linear accelerator equipped with a beam modulator multileaf collimator. Set-up and isocenter position were controlled using daily cone-beam CT image guidance. The most common schemes of dose prescription were $24-30$ Gy in 3 fractions to $80 \%$ isodose. Dose constraints for organs at risk were: spinal cord maximum dose (Dmax) $<18$ Gy, heart Dmax $<30$ Gy, kidneys V15 Gy at $<35 \%$, trachea and main bronchi Dmax $<30$ Gy and esophagus Dmax <27 Gy (Figure 1).

\section{Statistical analysis}

Time to local failure was calculated from the date of first radiotherapy to the date of disease progression of each treated metastasis. The progression of a treated metastasis, within the high dose region of radiotherapy, was defined radiographically according to RECIST criteria modified for thymic epithelial tumors (14). Patient without evidence of disease progression in a treated target were censored. Progression free survival (PFS) was calculated from the date of the first radiation treatment to the date of relapse or death for any cause. Patients without evidence of tumor progression were censored if still alive at the time of data analysis. Kaplan-Meier curves were generated and differences analyzed using the Log-Rank test. All tests were considered statistically significant for $\mathrm{P}<0.05$ and performed using SPSS version 21 (SPSS Inc., Chicago, IL, USA).

\section{Results}

From January 2013 to December 2018, 22 patients with thymoma received SBRT for the treatment of their pleural metastases at our institutions. Patients' characteristics 
Table 1 Patient characteristics

\begin{tabular}{|c|c|}
\hline Characteristics & $\mathrm{N}=22$ \\
\hline Age (years), median (range) & 40 (29 to 73$)$ \\
\hline \multicolumn{2}{|l|}{ Sex } \\
\hline Male & 12 \\
\hline Female & 10 \\
\hline \multicolumn{2}{|l|}{ ECOG PS } \\
\hline 0 & 10 \\
\hline 1 & 12 \\
\hline \multicolumn{2}{|l|}{ WHO histology } \\
\hline$A$ & 1 \\
\hline$A B$ & 3 \\
\hline B1 & 3 \\
\hline B2 & 3 \\
\hline B2/B3 & 3 \\
\hline B3 & 9 \\
\hline \multicolumn{2}{|l|}{ Stage at the diagnosis } \\
\hline IIA & 2 \\
\hline IIB & 7 \\
\hline III & 5 \\
\hline IVA & 7 \\
\hline IVB & 1 \\
\hline
\end{tabular}

WHO histology: classification of thymoma according to 2015 World Health Organization classification schema; stage at the diagnosis: according to Masaoka and Koga staging system. ECOG PS, Eastern Cooperative Oncology Group Performance Status.

are summarized in Table 1. Median follow-up was 33.2 months (95\% CI: 13.1-53.3 months). At the time of the diagnosis, 7 patients had already pleural metastases (stage IVA) and 1 patient had pleural and pulmonary metastases (stage IVB). Seven patients received chemotherapy before the resection. All patients, but one, underwent thymectomy for the resection of their primary tumor (Table 2). The patient with pulmonary and pleural metastases had a B3 thymoma and underwent resection after preoperative chemotherapy. A patient with a stage IVA B3 thymoma did not receive cytoreductive surgery but only chemotherapy. After 2 years this patient experienced progression of a singular pleural metastasis that was treated with SBRT. After resection of the primary tumor, 12 patients received
Table 2 Tumor characteristics and treatments

\begin{tabular}{|c|c|}
\hline Terapeutic procedure & Value \\
\hline \multicolumn{2}{|l|}{ Preoperative chemotherapy } \\
\hline Yes & 7 \\
\hline No & 15 \\
\hline \multicolumn{2}{|l|}{ Thymectomy } \\
\hline Yes & 21 \\
\hline No & 1 \\
\hline \multicolumn{2}{|l|}{ Postoperative radiotherapy to the anterior mediastinum } \\
\hline Yes & 12 \\
\hline No & 10 \\
\hline \multicolumn{2}{|l|}{ Resection of pleural metastases } \\
\hline Yes & 14 \\
\hline No & 8 \\
\hline \multicolumn{2}{|l|}{ Pleural metastases at the time of radiotherapy } \\
\hline Single & 5 \\
\hline Multiple & 17 \\
\hline \multicolumn{2}{|l|}{ Extra pleural metastases before radiotherapy } \\
\hline Yes & 3 \\
\hline No & 19 \\
\hline $\begin{array}{l}\text { Number of treated metastases with SBRT in each } \\
\text { patient, median (range) }\end{array}$ & 3 (1 to 10$)$ \\
\hline \multicolumn{2}{|l|}{ Radiation dose } \\
\hline 30 Gy in 3 fractions & 41 \\
\hline 24 Gy in 3 fractions & 19 \\
\hline 40 Gy in 5 fractions & 8 \\
\hline
\end{tabular}

Postoperative radiotherapy was given to the anterior mediastinum after resection of the primary tumor. Resection of pleural metastases indicates the number of patients who previously underwent a surgical resection of pleural metastases; after a subsequent progression of pleural metastases, they received radiotherapy. SBRT, stereotactic body radiation therapy.

postoperative radiotherapy with 50 Gy in 25 fractions on the anterior mediastinum. Fourteen patients underwent surgery for pleural metastases before SBRT. Seven patients received a wide local excision, 3 patients a wide local excision with hyperthermic intrathoracic chemotherapy (HITHOC), 1 patient palliative pleurectomy with HITHOC and 3 patients pleurectomy/decortication with HITHOC. Eight patients received a second surgery after pleural progression 

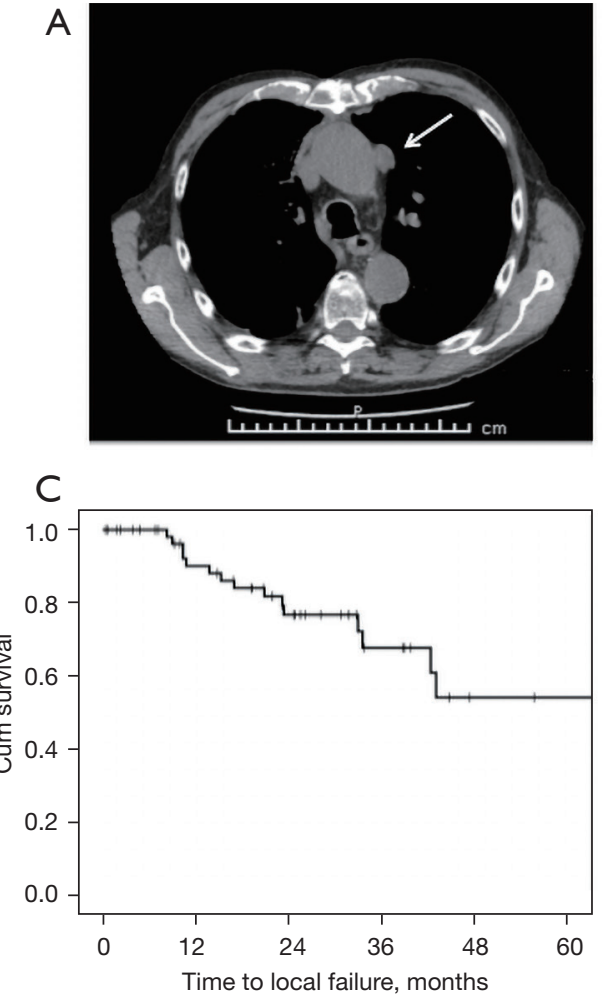

B

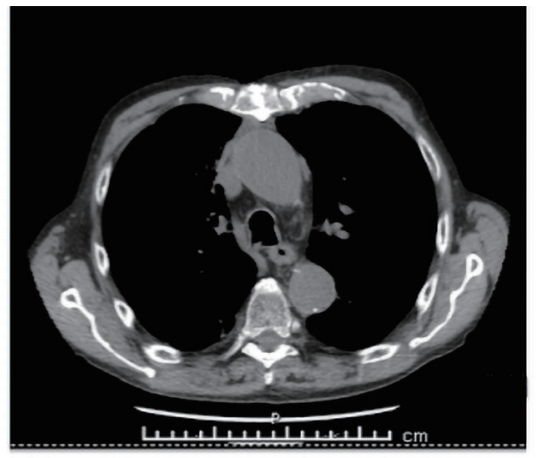

D

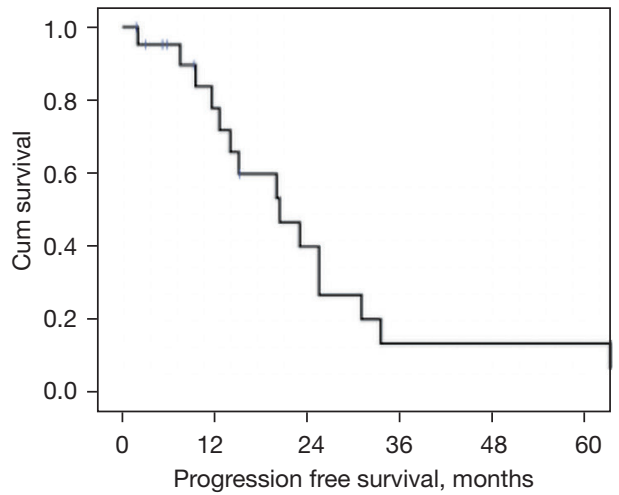

Figure 2 Thymoma metastasis in the left mediastinal pleura (arrow) before (A) and (B) after radiotherapy. Kaplan Maier curves of time to local failure (C) and progression free survival (D).

before SBRT: 4 wide local excisions, 2 wide local excisions with HITHOC and 1 pleurectomy/decortication with HITHOC. Unfortunately, this kind of surgery is a palliative treatment and patients experienced further evidences of pleural progression for which radiosurgery become an option. In the remaining 8 patients, SBRT was preferred to surgery because of patients' clinical condition or refusal of an invasive approach. Five patients had a single pleural metastasis and 17 had multiple localizations (from 2 to 10 pleural sites). Sixteen patients received SBRT on multiple sites of pleural metastases with a median of 3 and a range from 2 to 10. At the time of the analysis a patient received SBRT exclusively on 1 of its 3 pleural metastases. A total of 68 pleural metastases have been treated using SBRT. The objective response rate was $54.5 \%$ with 4 complete and 8 partial responses (Figure $2 A, 2 B$ ). Fifteen treated metastases underwent progression in 10 patients. The median time to local failure was not reached, with a local disease control rate of $92 \%, 78 \%$ and $71 \%$ after 1,2 and 3 years, respectively (Figure 2C). At the time of data analysis, 16 patients experienced disease progression, with a median
PFS of 20.4 months (95\% CI: 10.7-30.0 months; Figure 2D). Disease control rate was $79 \%, 41 \%$ and $14 \%$ after 1,2 and 3 years, respectively. There were not significant differences in PFS between patients diagnosed with synchronous and metachronous metastases (median PFS 23.0 and 20.0 months, respectively; log-rank $\mathrm{P}=0.477$ ), between those treated or not with chemotherapy for metastatic disease (median PFS 25.5 and 20.0 months, respectively; $\log$-rank $\mathrm{P}=0.189$ ) and between those who received or not a surgical resection of the pleural metastases before SBRT (median PFS 20.4 and 20.0 months, respectively, log-rank $\mathrm{P}=0.871)$. There were not significant differences in PFS $(\mathrm{P}=0.516)$ between $\mathrm{A}-\mathrm{AB}-\mathrm{B} 1$ and $\mathrm{B} 2-\mathrm{B} 3$ histotypes. Followup was not mature for overall survival estimation at the time of data analysis. We did not observe grade (G)3-4 toxicity related to the treatment. After SBRT, 4 patients had G1 cough, one patient had G1 hiccups, 2 patients had G2 voice change, 4 patients had transient G1 pleuritic pain, and 5 patients had G1 actinic pneumonitis in proximity of the treated area. One patient had rib fractures related to the treatment. A patient developed aplastic anemia after 
2 months from the treatment and required blood transfusions and immunosuppressive treatment. Nineteen patients with myasthenia gravis and 3 with neuromyotonia were receiving corticosteroids at the time of SBRT. None of them showed a worsening of neuromuscular symptoms within 6 months from SBRT.

\section{Discussion}

In our series of thymoma patients, the treatment of pleural metastases with SBRT is feasible without relevant toxicity and can yield a satisfactory local control of the disease.

Thymomas are reputed chemotherapy sensitive tumors. In chemotherapy naïve patients, objective response rate ranges between $50 \%$ and $92 \%$ for schedule containing cisplatin and doxorubicin with a median PFS between 9.5 and 18.4 months $(15,16)$. After progression, second line chemotherapy is less effective with response rates ranging between $10 \%$ and $40 \%$ and a median PFS between 7.6 and 11.3 months (17-19). Targeted drugs, including sunitinib and pembrolizumab, obtained promising results in thymic carcinomas but appear less effective in thymomas. Immunotherapy significantly increases the risk of immune-related paraneoplastic syndromes in thymomas. In this scenario, local treatment of oligometastatic or oligoprogressive patients becomes an appealing option. The pleural cavity is the most common site of metastatic diffusion in thymomas (20). Surgery is the mainstay of treatment for localized tumors. After resection, 10-year disease-free survival is $94 \%, 88 \%, 56 \%$ and $33 \%$ for stage I, II, III and IVa thymoma, respectively (21). The chance to carry out a complete resection is the most important prognostic factor. WHO histotype is an independent prognostic factor in some series (21). The most common site of relapse is the pleura: $54.1 \%$ to $58.1 \%$ (20). In these patients, the resection of pleural metastases can be taken into account depending on their characteristics: homolateral, the number of lesions, their dimension and the infiltration of surrounding structures. Pleural and pericardial metastases can be present already at the time of the diagnosis in $5-10 \%$ of thymic epithelial tumors and define the stage IVA according to the Masaoka and Koga staging system (22). B2 and $\mathrm{B} 3$ thymomas present a higher chance to have pleural metastases at the time of diagnosis respect to $\mathrm{A}, \mathrm{AB}$ and $\mathrm{B} 1$ histotypes (22). Depending on the number and dimensions of pleural metastases, different surgical approaches can be considered, including: extrapleural pneumonectomy, total pleurectomy, pleurectomy/decortication and local pleurectomy (23). During surgery, the use of intrapleural chemotherapy and hyperthermia remains controversial for the absence of randomized clinical trials. Despite the absence of conclusive evidences, several authors adopt intrapleural chemotherapy and hyperthermia (20). Surgical resection of pleural metastases can control locally the diseases and can obtain long intervals without progression in selected patients (24). The thymic working group of the European society of thoracic surgery (ESTS) has described results of 152 patients, who received resection of pleural metastases. The median time to recurrence after the first pleural surgery was 46 months with $87.2 \%$ of patients alive after 5 years from the resection. Interestingly, about $50 \%$ of patients who progressed had a relapse in the pleural cavity. In these patients a redo strategy can be performed in selected cases but repeatability of surgery is limited. Indeed, surgery is invasive and is not feasible in all patients because of comorbidities and technical challenges. Outcome of surgical series suggests that tumor progression remains limited to the pleural cavity in many thymomas, therefore, these cases could benefit of local treatments in addiction to systemic drugs. Advances in radiotherapy technologies allow the treatment of tumor lesions with radical doses and limited toxicity to surrounding organs. Despite the sensitivity of thymoma to radiation, the use of radiotherapy in pleural metastasis remains barely investigated to date. Appling SBRT to pleural metastases, we obtained a promising objective response rate and only 15 treated metastases progressed with a local disease control rate of $92 \%, 78 \%$ and $71 \%$ after 1,2 and 3 years, respectively. In stage I non-small cell lung cancer (NSCLC) patients, SBRT is the treatment of choice for not operable patients. In stage I NSCLC, the 3-year local control rate ranges between 79.6 and $97.6 \%$ (25-27). Compared to stage I NSCLC, we observed a lower 3-year disease control rate (71\%). Moreover, SBRT has been used for the treatment of oligometastatic NSCLC, frequently in combination with chemotherapy and surgery. In oligometastatic NSCLC, SBRT achieved a disease control rate of 77-92\% (28-31). Our data are more similar to these results. Indeed, patients included in our analysis are metastatic and 17 of them have multiple pleural localizations. The anatomy of pleural cavity could favor the progression of treated metastases reseeding of cancer cells through the pleural fluid. Indeed, in stage I NSCLC patients treated with SBRT, the contact of the tumor with the pleura reduces the chance of survival (32). The comparison of local disease control in thymoma and NSCLC should be carefully considered because thymoma 
patients have an extended life expectancy, compared to NSCLC. In our series only three patients have died at the time of data analysis. On the contrary, a significant proportion of NSCLC patients died within few months from the treatment, limiting the chance to develop a progression of treated lesions.

The median PFS was 20.4 months with 16 patients experiencing a disease progression at the time of data analysis. The comparison of the observed PFS with those of other reports could be misleading because of the retrospective nature of our report with several possible selection bias including thymoma histotype. We did not observe any G3-4 toxicities with SBRT with only one patient with costal bone fracture and none symptomatic pneumonitis or pleural effusion. Toxicity was mainly related to local inflammation nearby the radiotherapy filed. The development of aplastic anemia could be related to the treatment since it occurred within 2 months from the SBRT but a link with the progression of thymoma can not be excluded. Therefore, we believe this treatment is feasible in thymoma patients with pleural metastases and a prospective trail could be useful to confirm our hypothesis.

Surgery remains the first option for the treatment of pleural metastases of thymoma. Our data suggest that SBRT could be an additional option for the treatment of patients not eligible for surgical resection, for patients who refuse surgery and for those not technically resectable anymore after one or multiple surgical resections. Patients with an extensive tumor load or with a fast cancer growth remain candidate for chemotherapy.

\section{Acknowledgments}

Funding: None.

\section{Footnote}

Conflicts of Interest: All authors have completed the ICMJE uniform disclosure form (available at https://dx.doi. org/10.21037/jtd-19-3799). The authors have no conflicts of interest to declare.

Ethical Statement: The authors are accountable for all aspects of the work in ensuring that questions related to the accuracy or integrity of any part of the work are appropriately investigated and resolved. The study was conducted in accordance with the Declaration of Helsinki (as revised in 2013). The study was approved from our institution ethics committee CEAVNO with number ID 15960 and individual consent for this retrospective analysis was waived.

Open Access Statement: This is an Open Access article distributed in accordance with the Creative Commons Attribution-NonCommercial-NoDerivs 4.0 International License (CC BY-NC-ND 4.0), which permits the noncommercial replication and distribution of the article with the strict proviso that no changes or edits are made and the original work is properly cited (including links to both the formal publication through the relevant DOI and the license). See: https://creativecommons.org/licenses/by-nc-nd/4.0/.

\section{References}

1. de Jong WK, Blaauwgeers JL, Schaapveld M, et al. Thymic epithelial tumours: a population-based study of the incidence, diagnostic procedures and therapy. Eur J Cancer 2008;44:123-30.

2. Marx A, Chan JK, Coindre JM, et al. The 2015 World Health Organization Classification of Tumors of the Thymus: Continuity and Changes. J Thorac Oncol 2015;10:1383-95.

3. Kelly RJ, Petrini I, Rajan A, et al. Thymic malignancies: from clinical management to targeted therapies. J Clin Oncol 2011;29:4820-7.

4. Petrini I, Meltzer PS, Kim IK, et al. A specific missense mutation in GTF2I occurs at high frequency in thymic epithelial tumors. Nat Genet 2014;46:844-9.

5. Eng TY, Fuller CD, Jagirdar J, et al. Thymic carcinoma: state of the art review. Int J Radiat Oncol Biol Phys 2004;59:654-64.

6. Attaran S, Acharya M, Anderson JR, et al. Does surgical debulking for advanced stages of thymoma improve survival? Interact Cardiovasc Thorac Surg 2012;15:494-7.

7. Hamaji M, Kojima F, Omasa M, et al. A meta-analysis of debulking surgery versus surgical biopsy for unresectable thymoma. Eur J Cardiothorac Surg 2015;47:602-7.

8. Lucchi M, Davini F, Ricciardi R, et al. Management of pleural recurrence after curative resection of thymoma. J Thorac Cardiovasc Surg 2009;137:1185-9.

9. Lucchi M, Basolo F, Mussi A. Surgical treatment of pleural recurrence from thymoma. Eur J Cardiothorac Surg 2008;33:707-11.

10. Videtic GMM, Donington J, Giuliani M, et al. Stereotactic body radiation therapy for early-stage non-small cell lung cancer: Executive Summary of an ASTRO Evidence-Based 
Guideline. Pract Radiat Oncol 2017;7:295-301.

11. Timmerman R, Heinzerling J, Abdulrahman R, et al. Stereotactic body radiation therapy for thoracic cancers: recommendations for patient selection, setup and therapy. Front Radiat Ther Oncol 2011;43:395-411.

12. Hao XJ, Peng B, Zhou Z, et al. Prospective Study of Stereotactic Body Radiation Therapy for Thymoma and Thymic Carcinoma: Therapeutic Effect and Toxicity Assessment. Sci Rep 2017;7:13549.

13. Detterbeck FC, Nicholson AG, Kondo K, et al. The Masaoka-Koga stage classification for thymic malignancies: clarification and definition of terms. J Thorac Oncol 2011;6:S1710-6.

14. Benveniste MF, Korst RJ, Rajan A, et al. A practical guide from the International Thymic Malignancy Interest Group (ITMIG) regarding the radiographic assessment of treatment response of thymic epithelial tumors using modified RECIST criteria. J Thorac Oncol 2014;9:S119-24.

15. Loehrer PJ Sr, Kim K, Aisner SC, et al. Cisplatin plus doxorubicin plus cyclophosphamide in metastatic or recurrent thymoma: final results of an intergroup trial. The Eastern Cooperative Oncology Group, Southwest Oncology Group, and Southeastern Cancer Study Group. J Clin Oncol 1994;12:1164-8.

16. Fornasiero A, Daniele O, Ghiotto C, et al. Chemotherapy for invasive thymoma. A 13-year experience. Cancer 1991;68:30-3.

17. Bonomi PD, Finkelstein D, Aisner S, et al. EST 2582 phase II trial of cisplatin in metastatic or recurrent thymoma. Am J Clin Oncol 1993;16:342-5.

18. Palmieri G, Merola G, Federico P, et al. Preliminary results of phase II study of capecitabine and gemcitabine (CAP-GEM) in patients with metastatic pretreated thymic epithelial tumors (TETs). Ann Oncol 2010;21:1168-72.

19. Gbolahan OB, Porter RF, Salter JT, et al. A Phase II Study of Pemetrexed in Patients with Recurrent Thymoma and Thymic Carcinoma. J Thorac Oncol 2018;13:1940-8.

20. Roden AC, Molina JR. Editorial: pleural recurrence of thymoma-what is the value of intra-thoracic chemohyperthermia? J Thorac Dis 2017;9:3583-6.

21. Detterbeck FC, Parsons AM. Management of stage I and II thymoma. Thorac Surg Clin 2011;21:59-67, vi-vii.

22. Kondo K, Monden Y. Therapy for thymic epithelial tumors: a clinical study of 1,320 patients from Japan. Ann Thorac Surg 2003;76:878-84; discussion 884-5.

23. Moser B, Fadel E, Fabre D, et al. Surgical therapy of thymic tumours with pleural involvement: an ESTS Thymic Working Group Project. Eur J Cardiothorac Surg
2017;52:346-55.

24. Yellin A, Simansky DA, Ben-Avi R, et al. Resection and heated pleural chemoperfusion in patients with thymic epithelial malignant disease and pleural spread: a single-institution experience. J Thorac Cardiovasc Surg 2013;145:83-7; discussion 87-9.

25. Guckenberger M, Allgäuer M, Appold S, et al. Safety and efficacy of stereotactic body radiotherapy for stage 1 non-small-cell lung cancer in routine clinical practice: a patterns-of-care and outcome analysis. J Thorac Oncol 2013;8:1050-8.

26. Ricardi U, Frezza G, Filippi AR, et al. Stereotactic Ablative Radiotherapy for stage I histologically proven non-small cell lung cancer: an Italian multicenter observational study. Lung Cancer 2014;84:248-53.

27. Timmerman R, Paulus R, Galvin J, et al. Stereotactic body radiation therapy for inoperable early stage lung cancer. JAMA 2010;303:1070-6.

28. Collen C, Christian N, Schallier D, et al. Phase II study of stereotactic body radiotherapy to primary tumor and metastatic locations in oligometastatic nonsmall-cell lung cancer patients. Ann Oncol 2014;25:1954-9.

29. Iyengar P, Wardak Z, Gerber DE, et al. Consolidative Radiotherapy for Limited Metastatic Non-Small-Cell Lung Cancer: A Phase 2 Randomized Clinical Trial. JAMA Oncol 2018;4:e173501.

30. Ouyang $W$, Yu J, Nuerjiang S, et al. Stereotactic body radiotherapy improves the survival of patients with oligometastatic non-small cell lung cancer. Cancer Med 2019;8:4605-14.

31. De Ruysscher D, Wanders R, van Baardwijk A, et al. Radical treatment of non-small-cell lung cancer patients with synchronous oligometastases: long-term results of a prospective phase II trial (Nct01282450). J Thorac Oncol 2012;7:1547-55.

32. Eriguchi T, Takeda A, Tsurugai Y, et al. Pleural contact decreases survival in clinical T1N0M0 lung cancer patients undergoing SBRT. Radiother Oncol 2019;134:191-8.

Cite this article as: Pasquini G, Menichelli C, Pastore G, Casamassima F, Fabrini MG, Cappelli S, Valleggi S, Lucchesi M, Lucchi M, Ricciardi R, Maestri M, Guida M, Chella A, Petrini I. Stereotactic body radiation therapy for the treatment of pleural metastases in patients with thymoma: a retrospective review of 22 patients. J Thorac Dis 2021;13(11):6373-6380. doi: 10.21037/ jtd-19-3799 